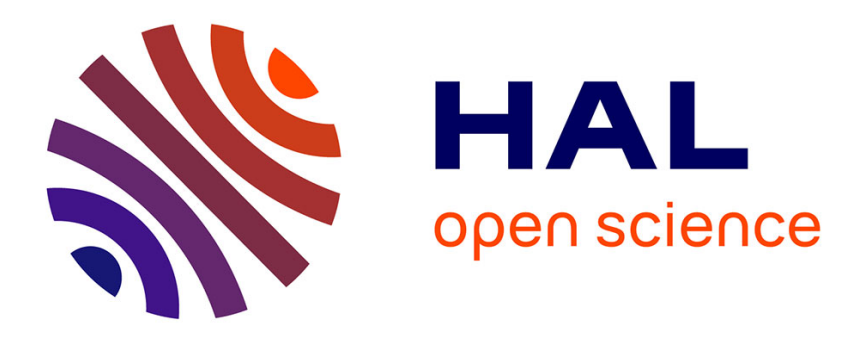

\title{
Applications des lasers à l'étude de l'atmosphère
}

\author{
G. Megie
}

\section{To cite this version:}

G. Megie. Applications des lasers à l'étude de l'atmosphère. Revue de Physique Appliquée, 1979, 14

(2), pp.369-378. 10.1051/rphysap:01979001402036900 . jpa-00244605

\section{HAL Id: jpa-00244605 https://hal.science/jpa-00244605}

Submitted on 1 Jan 1979

HAL is a multi-disciplinary open access archive for the deposit and dissemination of scientific research documents, whether they are published or not. The documents may come from teaching and research institutions in France or abroad, or from public or private research centers.
L'archive ouverte pluridisciplinaire HAL, est destinée au dépôt et à la diffusion de documents scientifiques de niveau recherche, publiés ou non, émanant des établissements d'enseignement et de recherche français ou étrangers, des laboratoires publics ou privés. 


\title{
Applications des lasers à l'étude de l'atmosphère
}

\author{
G. Megie \\ (Reçu le 30 juin 1978, révisé le 17 novembre 1978, accepté le 20 novembre 1978)
}

\begin{abstract}
Résumé. - L'utilisation des lasers en physique atmosphérique a permis d'abaisser le seuil de détection d'un grand nombre de composés minoritaires. On étudie les principes généraux du système lidar et les processus physiques d'interaction entre la lumière et le gaz atmosphérique et l'on décrit les principaux résultats obtenus entre le sol et $100 \mathrm{~km}$ d'altitude. Les limites actuelles des différentes méthodes ainsi que leurs perspectives d'avenir sont également mises en évidence.
\end{abstract}

Abstract. - The use of laser systems in geophysics increases the sensibility of the optical methods to detect minor constituents of the atmosphere. The principles of the lidar technique and the interaction processes between light and atmospheric matter are reviewed together with the main results obtained between the ground and $100 \mathrm{~km}$. The present limitations of the different methods and their future applications are also studied.

Les observations dans le domaine des longueurs d'onde optiques ont toujours contribué de façon importante à une meilleure connaissance de l'atmosphère terrestre : les phénomènes lumineux comme les aurores ou la luminescence du ciel sont restés jusqu'au milieu de ce siècle les seules manifestations accessibles de la haute atmosphère. Les principaux constituants atmosphériques ont ainsi été mis en évidence grâce à l'étude de l'absorption du flux solaire, directe ou diffusée au moyen de spectrographes. L'utilisation plus récente de moyens spatiaux, fusées, satellites ou ballons, a permis d'améliorer ces techniques d'observation, de réaliser des mesures in situ et d'accéder aux composés très minoritaires de la stratosphère ou de la mésosphère. Mais toutes ces techniques présentent l'inconvénient, soit d'être tributaires d'une source de rayonnement extérieure, et donc incontrôlable, le soleil, soit de ne pouvoir être utilisées que pour des mesures ponctuelles ne permettant pas une surveillance continue.

Déjà, avant même l'existence des lasers, l'extension des techniques radar aux longueurs d'ondes optiques avait été tentée avec des sources conventionnelles [1]. Mais, la découverte du laser dans les années 1960, et les nombreux développements qui ont conduit vers 1967 à la mise au point de lasers à fréquences accordables dans les domaines visible et infrarouge ont ouvert un nouveau champ de recherches pour l'optique atmosphérique.

Les propriétés des lasers qui rendent leur utilisation dans l'atmosphère attrayante sont, d'une part, les caractéristiques spatiales (directivité et possibilité d'émettre des pulses très courts), ce qui permet d'effec- tuer des sondages à distance avec une grande résolution spatiale, et d'autre part, leurs caractéristiques spectrales qui grâce à une grande monochromaticité permettent d'obtenir des densités spectrales de puissance supérieures de plusieurs ordres de grandeur à celles de la source solaire. La possibilité d'accord en fréquence permet enfin d'envisager des méthodes d'observation utilisant l'excitation ou l'absorption sélective par des constituants atmosphériques spécifiques. Il serait d'ailleurs vain de prétendre couvrir ici l'ensemble des applications des lasers aux études atmosphériques et, en particulier, nous n'envisageons pas les possibilités liées aux mesures télémétriques proprement dites dans le domaine de la géodésie ou de la physique des nuages. Nous étudierons surtout les principes généraux du système lidar et les processus physiques d'interaction entre la lumière et le gaz, ainsi que quelques exemples de réalisation dans les différents domaines de l'atmosphère entre le sol et les hautes altitudes $(100 \mathrm{~km})$ à la limite de la thermosphère.

1. Principe du système lidar. - Le principe du système lidar (ou radar optique) est la transposition directe dans le domaine des longueurs d'ondes optiques de celui du radar : il consiste à mesurer l'intensité d'un signal rétrodiffusé en fonction du temps de parcours de la lumière dans son trajet aller-retour entre le système émetteur-récepteur et la zone diffusante. Dans sa version la plus classique (Fig. 1), l'émetteur est un laser pulsé dont la durée de pulse définit la résolution spatiale ultime dans l'axe du faisceau. La divergence du laser nécessite en général l'utilisation 


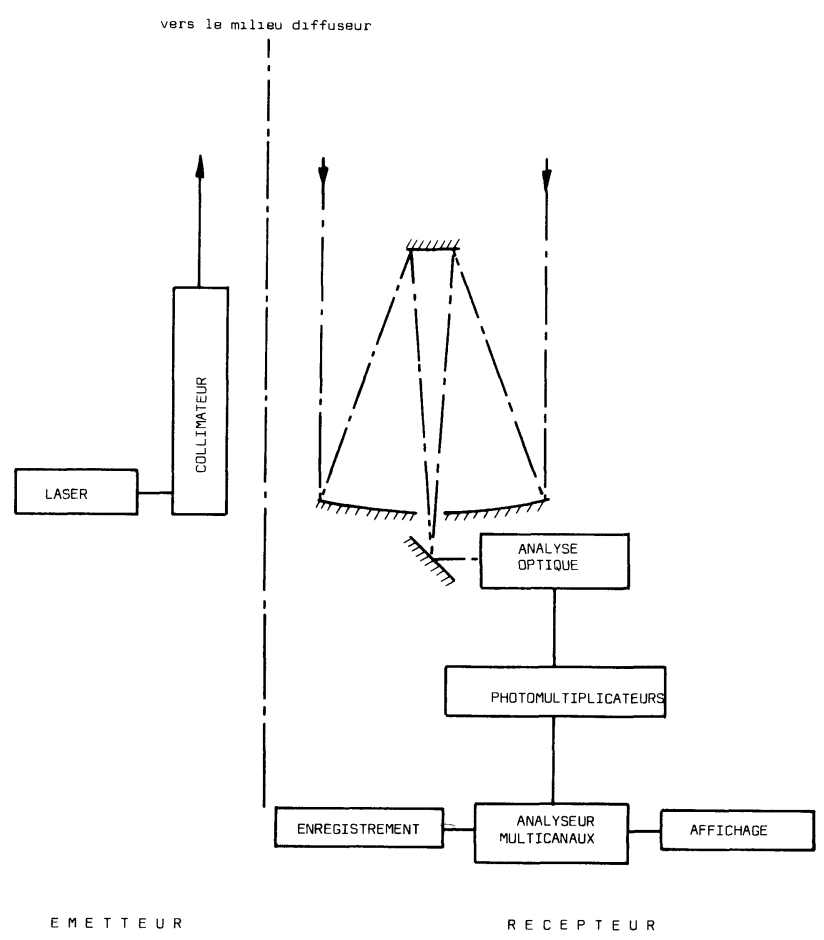

Fig. 1. - Schéma de principe du système lidar.

à l'émission, d'une lunette collimatrice afin d'obtenir des valeurs acceptables, de l'ordre de quelques dixièmes de milliradians, et même inférieures pour les observations de jour. Les photons rétrodiffusés sont reçus sur un télescope qui peut ou non être placé au même point que le laser émetteur (montage monostatique ou bistatique) et, après passage dans un filtre interférentiel étroit qui élimine le signal parasite dû au fond du ciel, focalisés sur un détecteur adapté à la longueur d'onde de réception (photomultiplicateur ou cellule infrarouge). Une chaîne électronique couplée par un système logique au laser émetteur fait l'analyse temporelle de l'écho, de façon à en restituer la répartition spatiale. Les données sont en général enregistrées simultanément sur bande magnétique et visualisées en temps réel sur un écran d'oscilloscope ou un enregistreur graphique.

Afin de préciser les différentes méthodes de mesure associées au système lidar, on peut considérer l'équation simplifiée qui permet d'obtenir la puissance rétrodiffusée en fonction des paramètres du système :

$$
\begin{gathered}
P(z, z+\mathrm{d} z)=P_{0} \times G(z) \times \tau_{\mathrm{n}}(z, z+\mathrm{d} z) \\
\times \mathrm{e}^{-2 \tau_{\mathbf{a}}(0, z+\mathrm{d} z)}
\end{gathered}
$$

où $P$ représente la puissance moyenne reçue à partir de l'élément diffuseur d'altitude comprise entre $z$ et $z+\mathrm{d} z$

$P_{0}$ la puissance moyenne émise par le laser :

$G(z)$ est un facteur lié à la géométrie du système qui varie en raison inverse de $z^{2}$, distance de l'émetteur à la zone diffusante et qui inclut le rendement des différents composants optiques ou photo-électriques ; $\tau_{\mathbf{n}}(z, z+\mathrm{d} z)$ représente l'épaisseur optique de l'élément diffusant à la longueur d'onde d'émission et peut donc s'écrire comme le produit :

$\tau_{\mathbf{n}}=\left(\sum_{\mathbf{i}} n_{\mathbf{i}} \sigma_{\mathbf{i}}\right) \cdot \mathrm{d} z$

de la résolution spatiale $\mathrm{d} z$ du système par la concentration $n_{\mathrm{i}}$ et la section efficace $\sigma_{\mathrm{i}}$ de diffusion des différents constituants atmosphériques responsables de l'écho.

Enfin, $\tau_{\mathrm{a}}(0, z+\mathrm{d} z)$ représente de la même façon l'épaisseur optique intégrée de l'atmosphère entre le sol et la zone diffusante.

Nous voyons ainsi que les concentrations caractéristiques des constituants atmosphériques peuvent être obtenues de deux façons différentes suivant l'importance relative des termes $\tau_{\mathrm{n}}$ et $\tau_{\mathrm{a}}$ : la mesure de $\tau_{\mathbf{n}}$ fait appel à la diffusion par le gaz atmosphérique ou par un constituant spécifique de la lumière laser, alors que pour obtenir $\tau_{\mathrm{a}}$, on doit considérer l'absorption par ces mêmes constituants; mais il est bien évident que pour une longueur d'onde donnée, les constituants susceptibles de diffuser les photons laser sont également ceux qui vont absorber à cette même longueur d'onde.

Pour mesurer un constituant i par rétrodiffusion, il est donc nécessaire que son épaisseur optique $\tau_{\mathrm{ni}}$ soit très supérieure à celle des autres constituants et plus particulièrement à celle du gaz atmosphérique et donc pour les composés minoritaires de l'atmosphère, que la longueur d'onde laser corresponde à une transition atomique ou moléculaire de l'espèce étudiée. Dans ce cas la précision obtenue sur la mesure de l'épaisseur optique du constituant $\mathrm{i}$ est directement reliée à l'erreur statistique sur le signal rétrodiffusé puisque $\frac{\mathrm{d} \tau_{\mathrm{n}}}{\tau_{\mathrm{n}}}=\frac{\mathrm{d} P}{P}$.

$\mathrm{Si}$ au contraire $\tau_{\mathrm{ni}}$ est très petit devant l'épaisseur optique du gaz atmosphérique, on utilisera l'absorption sélective du constituant $i$ en effectuant deux mesures laser à deux longueurs d'onde voisines $\lambda_{1}$ et $\lambda_{2}$ correspondant l'une à une transition du constituant $\mathrm{i}$, l'autre à un domaine de longueur d'onde sans absorption. On pourra alors déduire du rapport des puissances rétrodiffusées à $\lambda_{1}$ et $\lambda_{2}$ l'épaisseur optique intégrée du constituant étudié entre le sol et l'altitude $z+\mathrm{d} z$. Connaissant grâce à la résolution spatiale $\mathrm{du}$ système cette même épaisseur optique entre 0 et $z$, on a ainsi accès à la concentration moyenne du constituant i entre $z$ et $z+\mathrm{d} z$. C'est la technique d'absorption différentielle qui nécessite donc l'utilisation d'un système laser émettant à 2 longueurs d'onde.

Nous remarquerons également dans ce cas que l'élément diffusant atmosphérique peut être remplacé par un système réflecteur pour les mesures au sol, ce qui augmente la puissance de retour, mais évidemment élimine la résolution spatiale et ne permet donc plus d'obtenir que l'épaisseur optique intégrée du constituant étudié (Fig. 2). 

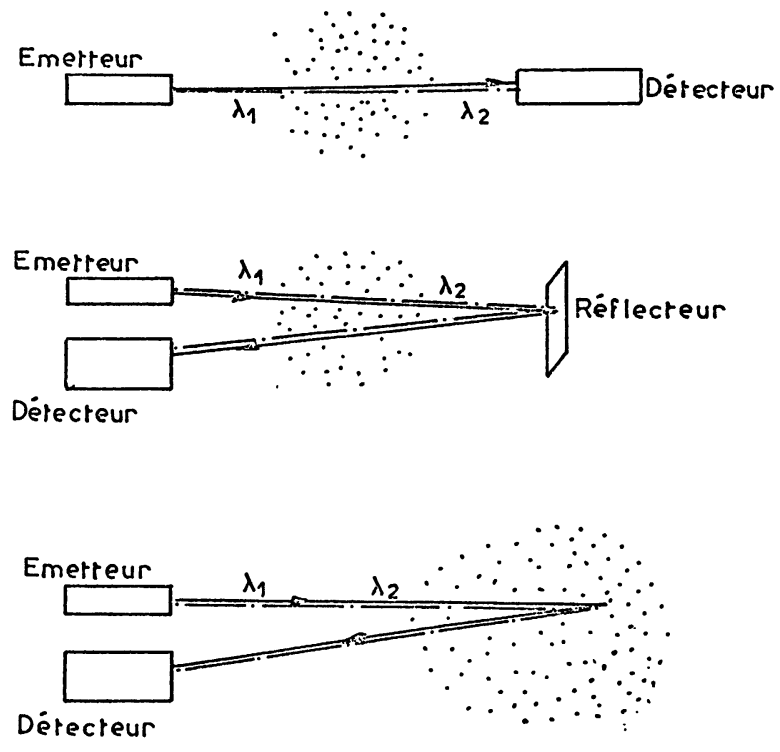

Fig. 2. - La technique lidar d'absorption différentielle.

La précision relative obtenue sur l'épaisseur optique et donc la concentration mesurée est donnée dans ce cas par

$\frac{\mathrm{d} \tau_{\mathrm{a}}}{\tau_{\mathrm{a}}}=\frac{1}{2 \tau_{\mathrm{a}}} \frac{\mathrm{d} P}{P}$

et nous voyons ainsi que pour des épaisseurs optiques à mesurer de l'ordre de $10^{-2}$ à $10^{-3}$, la précision relative sur le signal de retour est de 2 à 3 ordres de grandeurs supérieure à celle nécessaire dans le cas d'une simple rétrodiffusion.

Le facteur $G(z)$ est en général pour un télescope de dimension moyenne $(30 \mathrm{~cm}$ de diamètre) et pour une altitude de sondage de $20 \mathrm{~km}$ de l'ordre de $10^{-13}$ à $10^{-14}$. Pour une puissance émise de $1 \mathrm{~W}$ et une épaisseur optique $\tau_{\mathrm{n}}$ de l'ordre de $10^{-2}$ à $10^{-3}$, la puissance reçue est donc d'environ $10^{-15}$ à $10^{-17} \mathrm{~W}$, ce qui correspond à quelques dizaines de milliers de photo-électrons par seconde par élément diffuseur en haute altitude.

On peut également remarquer qu'entre une mesure effectuée à $1 \mathrm{~km}$ d'altitude et une mesure à $100 \mathrm{~km}$ d'altitude, la valeur signal varie avec $G(z)$ d'un facteur $10^{4}$. D'où la nécessité d'utiliser des systèmes de détection de sensibilités différentes suivant la zone étudiée afin, d'une part, de préserver la précision sur le signal de retour et, d'autre part, d'éviter les effets parasites générés par les signaux forts lors de la détection de signaux plus faibles [2].

On voit donc que le bilan de liaison de ce type d'expériences est extrêmement faible, d'où la nécessité de disposer d'un système émetteur suffisamment puissant tout en conservant les qualités spectrales et spatiales nécessaires pour une étude spectroscopique fine. Cette simultanéité dans les performances est souvent difficile à réaliser et la mise au point de systèmes laser pour les études atmosphériques constitue donc un sujet de recherches à part dans les techniques de développement laser qui doit intégrer les progrès les plus récents, à la fois dans le domaine des fortes énergies et dans celui de la pureté spectrale.

Nous n'avons d'ailleurs envisagé jusqu'ici que la mesure de concentrations à partir de l'intensité du signal rétrodiffusé. D'autres applications des lidars à la mesure de paramètres atmosphériques globaux sont basées sur l'étude des caractéristiques spectrales de ce signal. Ainsi la largeur spectrale de la raie rétrodiffusée par résonance optique d'un constituant considéré comme traceur peut conduire, dans le cas où l'élargissement est inhomogène (Doppler), à la mesure de la température de l'atmosphère neutre [3]. De même, une mesure du décalage en fréquence de la raie rétrodiffusée par rapport à sa valeur au sol permet en principe d'obtenir la vitesse du vent moyen dans la direction d'observation [4]. Mais ces mesures restent très difficiles et sont pour l'instant limitées en intérêt géophysique par leur précision même. Une étape importante sera franchie par la mise au point de lasers pulsés de puissance émettant sur des largeurs spectrales de quelques dizaines de $\mathrm{kHz}$, qui permettront ainsi d'effectuer une excitation sur une largeur inférieure à celle de la raie inhomogène et donc de transformer l'analyse spectrale de la raie rétrodiffusée en mesure différentielle d'intensité, avec un gain important de plusieurs ordres de grandeur sur la précision [5].

Nous allons maintenant passer en revue de façon plus détaillée ces différentes méthodes en montrant comment l'efficacité du processus étudié en détermine le domaine d'application et en donnant les principaux résultats expérimentaux obtenus et les perspectives d'avenir de chacune de ces méthodes.

2. Sondage par rétrodiffusion. - Dans ce cas, comme nous l'avons vu à partir de l'équation (1), c'est l'épaisseur optique $\tau_{n i}$ et donc le produit $n_{\mathbf{i}} \sigma_{\mathbf{i}}$ de la concentration du constituant étudié par la section efficace du processus diffusant qui détermine l'efficacité de la mesure.

2.1 Diffusion ÉlastiQue (Fig. $3 a$ ). - Nous pouvons tout d'abord remarquer que l'ensemble du gaz atmosphérique diffuse de façon élastique la lumière incidente et ce, quelle que soit la longueur d'onde utilisée, bien que l'efficacité de ce processus décroisse au fur

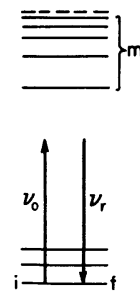

(a)

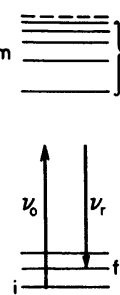

(b)

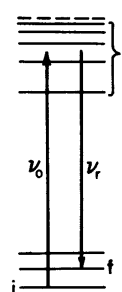

(c)

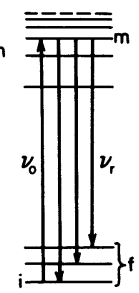

(d)

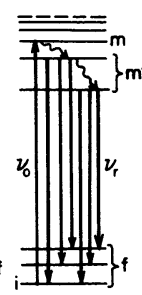

(e)

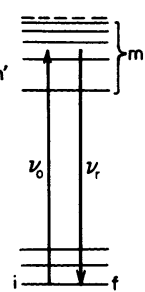

(f)
Fig. 3. - Processus d'interaction (d'après [57]) : a) diffusion Rayleigh; $b$ ) diffusion Raman; $c$ ) diffusion Raman résonnante ; $d$ ) résonance-fluorescence ; $e$ ) fluorescence $; f$ ) diffusion résonnante. 
et à mesure que la longueur d'onde augmente. On comprend ainsi que les premières mesures effectuées par sondage laser dans l'atmosphère aient été celles de la densité atmosphérique totale et ce d'autant plus, qu'elles ne nécessitaient pas une longueur d'onde spécifique pour le laser émetteur [6].

Si la longueur d'onde de la lumière incidente est très grande devant les dimensions caractéristiques des particules diffusantes, l'interaction se fait sans échanges appréciables d'énergie entre les photons et les atomes ou les molécules : c'est la diffusion Rayleigh dont les sections efficaces restent très faibles et varient comme $\lambda^{-4}$ entre $5 \times 10^{-26} \mathrm{~cm}^{2}$ à $300 \mathrm{~nm}$ et $4 \times 10^{-28} \mathrm{~cm}^{2}$ à $1 \mu \mathrm{m}$. Le choix de longueurs d'ondes courtes est donc en principe plus favorable. Mais si l'on tient compte des valeurs caractéristiques de la concentration atmosphérique totale qui varie entre $2 \times 10^{19} \mathrm{~cm}^{-3}$ au sol et $10^{13} \mathrm{~cm}^{-3}$ à $100 \mathrm{~km}$, on voit que pour une résolution en altitude de $1 \mathrm{~km}$, le rapport $P / P_{0}$ est de l'ordre de $10^{-20}$ aux hautes altitudes. D'où la nécessité pour ce type de mesures d'employer des lasers de très grande énergie par tir, ce qui explique que les mesures aient été en général effectuées avec des lasers à rubis et des télescopes de très grandes dimensions [7], [61]. Des profils de concentration totale ont ainsi été obtenus jusqu'au niveau de la basse thermosphère et les variations saisonnières ou de plus courtes périodes sous l'influence de mouvements atmosphériques globaux, tels que les marées ou les ondes de gravité ont ainsi été mises en évidence [8].

Dans le cas où les particules diffusantes ont des dimensions supérieures à $0,1 \mu$ comme les poussières, les particules d'aérosols ou les gouttelettes d'eau, le processus d'interaction est la diffusion de Mie pour laquelle les sections efficaces de diffusion sont très grandes $\left(\sim 10^{-10} \mathrm{~cm}^{2}\right.$ dans le domaine visible pour des particules de l'ordre de 0,1 à $1 \mu \mathrm{m}$ ).

Mais dans ce cas, les concentrations de particules observables au niveau troposphérique ou stratosphérique vont de quelques particules à quelques dizaines de particules par $\mathrm{cm}^{3}$, si bien que le bilan total est assez comparable à celui de la diffusion Rayleigh.

On doit en outre noter que pour une observation à une seule longueur d'onde, ces deux processus sont en fait indiscernables l'un de l'autre, si bien que l'épaisseur optique $\tau_{n}$ mesurée est la somme de deux termes, l'un dû à la diffusion par les atomes et les molécules de l'air, l'autre dû aux poussières en suspension. Si l'on tient compte du fait que la section efficace de diffusion Mie varie en première approximation comme $\lambda^{-1,5}$ (cette variation dépend à la fois de la taille, de l'indice et de la forme des particules) et donc différemment de la section efficace Rayleigh, on voit qu'une observation à deux longueurs d'onde distinctes permet de différencier en principe les deux contributions [9].

Les applications de la diffusion de Mie ont été largement utilisées en physique des aérosols et le lidar a déjà apporté des résultats importants dans le domaine des aérosols stratosphériques [10, 11, 12] (Fig. 4) et dans les variations observées de ces poussières lors d'éruptions volcaniques de première importance comme celle du Mont Aguun ou du Mont Fuego [13] : les particules étant alors injectées directement au niveau stratosphérique, l'évolution des concentrations en fonction du temps et de la distance au point d'injection peut conduire à des résultats importants quant à la circulation générale. Les sondages lidar ont également permis d'étudier la répartition spatiale en fonction du temps, des poussières libérées par les réacteurs des avions supersoniques au cours de leur vol stratosphérique [54].

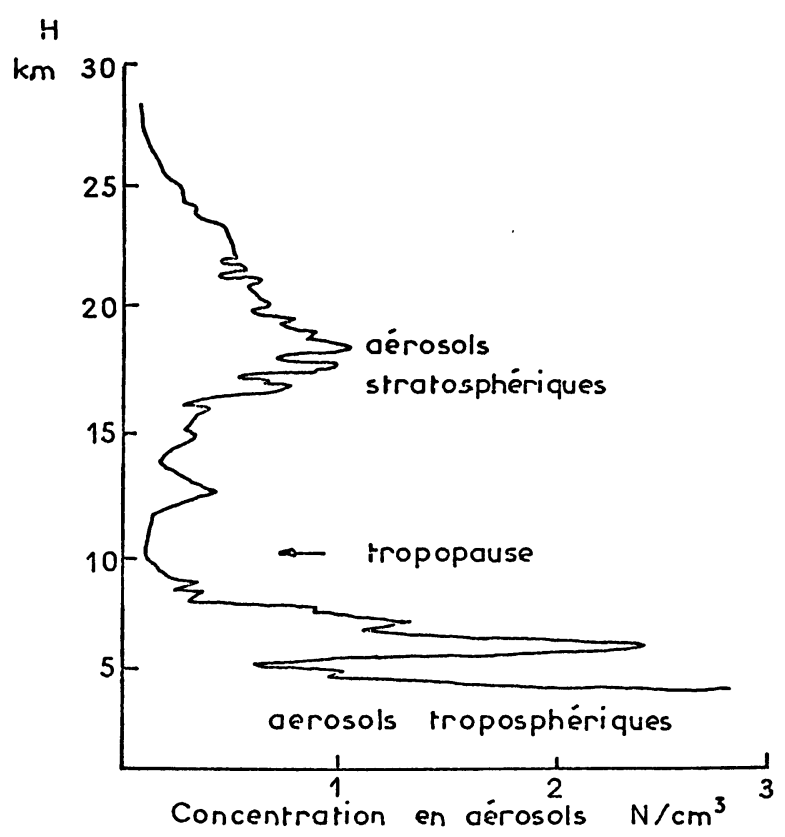

Fig. 4. - Profils verticaux des aérosols troposphériques et stratosphériques.

En physique des nuages, l'utilisation du lidar couplée à celle d'autres moyens de sondage à distance comme le radar Doppler permet d'obtenir des informations sur la densité de particules ou de noyaux de condensation et de corréler ces mesures à celles, par exemple, du rapport de mélange de la vapeur d'eau ou de la température $[14,15]$.

Les deux processus de diffusion élastique que nous venons d'évoquer ne permettent cependant pas une étude spécifique des différents constituants de l'atmosphère. Certes la mesure de l'ensemble des paramètres de Stokes [16] permet en principe de différencier les aérosols suivant leur nature ou leur taille, mais cela au prix d'une très grande complexité des mesures (plusieurs longueurs d'onde [17], polarisation [18] et de très nombreuses hypothèses théoriques). Nous allons maintenant envisager d'autres processus de diffusion inélastique et donc pour une longueur d'onde donnée, caractéristiques d'un seul constituant de l'atmosphère. 


\subsection{Processus de Diffusion inÉlastique. - Nous} distinguerons ici par ordre d'efficacité décroissante et donc pratiquement par ordre chronologique d'apparition dans les sondages laser de l'atmosphère : la diffusion résonnante, la fluorescence et la diffusion Raman.

- La diffusion résonnante (Fig. $3 f$ ) se produit lorsque la longueur d'onde d'émission de l'atome ou de la molécule est la même que la longueur d'onde excitatrice. Le temps d'interaction est de l'ordre de $10^{-8}$ à $10^{-9} \mathrm{~s}$ qui correspond à la durée de vie du niveau excité. Ce processus est très efficace puisque les sections efficaces varient de quelque $10^{-12} \mathrm{~cm}^{2}$ pour les atomes à $10^{-17} \mathrm{~cm}^{2}$ pour les molécules. Dès l'apparition des lasers à fréquence accordable, cette méthode a été utilisée pour étudier le sodium neutre qui existe à l'état d'atomes libres dans la haute atmosphère entre 80 et $100 \mathrm{~km}$ d'altitude $[19,20,21]$. Par opposition aux processus de diffusion élastique étudiés 2.1 et pour lesquels la largeur de la raie d'excitation peut atteindre quelques dixièmes de $\mathrm{nm}$, dans le cas de l'excitation d'une transition atomique, il est nécessaire de concentrer spectralement le maximum de photons sur une largeur utile de quelques pm : des développements importants de lasers à colorants pompés par flashs émettant à $589 \mathrm{~nm}$ avec de fortes densités spectrales ont ainsi été réalisés [22, 23], qui permettent d'obtenir couramment des énergies de l'ordre du Joule sur des largeurs spectrales inférieures à $8 \mathrm{pm}$.

Nous remarquerons que la section efficace du processus correspond en fait au produit de convolution de la raie laser et du profil spectral de la transition étudiée. On aura donc intérêt afin d'améliorer la précision des mesures à se placer, soit dans le cas d'une excitation constante sur le profil de raie et donc avec une largeur excitatrice de l'ordre de $10 \mathrm{pm}$, soit dans le cas d'une largeur d'excitation très fine devant la largeur spectrale de l'ordre de $0,1 \mathrm{pm}$. Pour des raisons évidentes de compatibilité entre les fortes puissances désirées et les largeurs spectrales possibles, c'est jusqu'ici la première solution qui a été retenue, les précisions obtenues sur les valeurs absolues des concentrations restant toutefois inférieures à $20 \%$. Mais l'apparition récente des techniques d'injection dans les lasers à colorants [24] permet d'envisager une excitation en bande plus fine $(<1 \mathrm{GHz})$ et donc spectralement plus efficace.

De la même façon et dans le même domaine d'altitude que pour le sodium, grâce à un laser à colorant (DOTC) pompé par un laser à rubis [25], on a pu étudier la concentration et le profil vertical du potassium, dont les concentrations $\left(\sim 100\right.$ atomes $\left.\mathrm{cm}^{-3}\right)$ sont pourtant inférieures d'un facteur 10 à 50 à celles du sodium. Cette étude simultanée des deux alcalins a ainsi contribué à la solution partielle du problème de leur origine [26] (Fig. 5).

On peut également noter que cette méthode de mesure est extrêmement sensible puisque des expé-

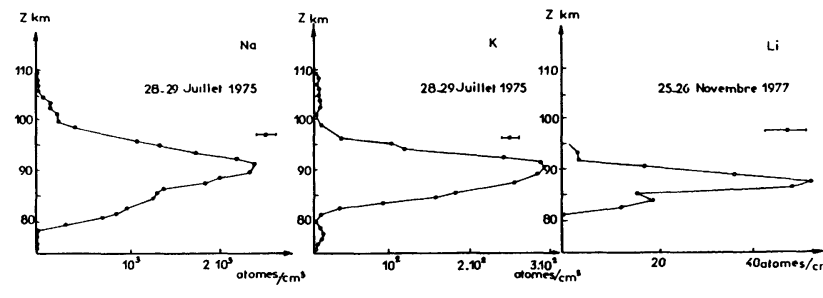

Fig. 5. - Profils verticaux du sodium, du potassium et du lithium atmosphériques.

riences récentes à $610 \mathrm{~nm}$ sur le lithium, autre constituant métallique de la haute atmosphère, ont permis de détecter à $90 \mathrm{~km}$ d'altitude des concentrations de l'ordre de 1 atome par $\mathrm{cm}^{3}$ (Fig. 5) [59].

Malheureusement les atomes libres n'existent en quantité importante dans l'atmosphère qu'aux altitudes supérieures à $50 \mathrm{~km}$ et, en ce qui concerne les principaux constituants comme l'oxygène ou l'hydrogène, les transitions de résonance sont situées dans l'ultraviolet lointain et donc inaccessible à partir du sol. Ce type d'études semble donc limité actuellement à l'étude de quelques constituants très particuliers et ne peut donner que des indications très indirectes sur le comportement global de l'atmosphère. C'est cependant, comme nous l'avons déjà mentionné, grâce à l'étude spectrale de la raie rétrodiffusée par le sodium qu'a pu être effectuée la première mesure directe à partir du sol, de la température de l'atmosphère neutre au niveau de la mésopause [27].

On peut cependant penser que l'extension des lasers dans le domaine ultraviolet (lasers à colorant doublés ou excimères) devrait permettre l'utilisation de cette méthode pour des mesures in situ des atomes majoritaires ou de radicaux libres de première importance dans l'atmosphère comme le radical $\mathrm{OH}$ [28].

- La fluorescence (Fig. 3d, e) correspond à une émission spontanée de photons à une longueur d'onde décalée vers le rouge par rapport à la longueur d'onde excitatrice. Les sections efficaces sont de l'ordre de quelque $10^{-18} \mathrm{~cm}^{2}$, mais la durée de vie des niveaux excités (de $1 \mu \mathrm{s}$ à $1 \mathrm{~s}$ ) est en général très supérieure au temps caractéristique du libre parcours moyen dans l'atmosphère, si bien que l'efficacité du processus est diminuée de plusieurs ordres de grandeur par les désactivations collisionnelles (quenching), surtout aux basses altitudes. On remarquera d'ailleurs qu'il en résulte une diminution de même importance de la durée de vie radiative du niveau excité, ce qui permet ainsi de retrouver une résolution spatiale fine. L'utilisation de cette méthode est en fait limitée aux altitudes supérieures (composants stratosphériques comme $\mathrm{NO}_{2}$ ou $\mathrm{OH}$ ), mais la nécessité de connaître avec précision les sections efficaces effectives rend l'interprétation des mesures très difficile [29].

- La diffusion Raman (Fig. $3 b$ ) résulte de l'excitation de transitions vibrationnelles ou rotationnelles des molécules. Le spectre Raman apparaît sous forme 
de raies diffusées, décalées par rapport à la longueur d'onde d'excitation d'une quantité caractéristique de la molécule étudiée. Un des avantages essentiels de ce processus est donc de pouvoir détecter simultanément plusieurs constituants à partir du même faisceau laser et d'obtenir ainsi une mesure précise des différents rapports de mélange par rapport à un constituant majoritaire comme l'azote (Fig. 6). D'autre part, le temps d'interaction court permet d'effectuer des mesures à toutes les altitudes. Cependant l'efficacité de ce processus reste faible puisque les sections efficaces de diffusion Raman sont inférieures de deux à trois ordres de grandeur aux sections efficaces de diffusion Rayleigh et correspondent à des valeurs de l'ordre de $10^{-30} \mathrm{~cm}^{2}$. La dépendance en longueur d'onde est analogue à celle observée dans le cas de la diffusion Rayleigh $\left(\sim \lambda^{-4}\right)$, ce qui justifie l'emploi de lasers émettant dans l'ultraviolet du type laser à rubis doublé ou lasers à azote $[30,31,60]$.
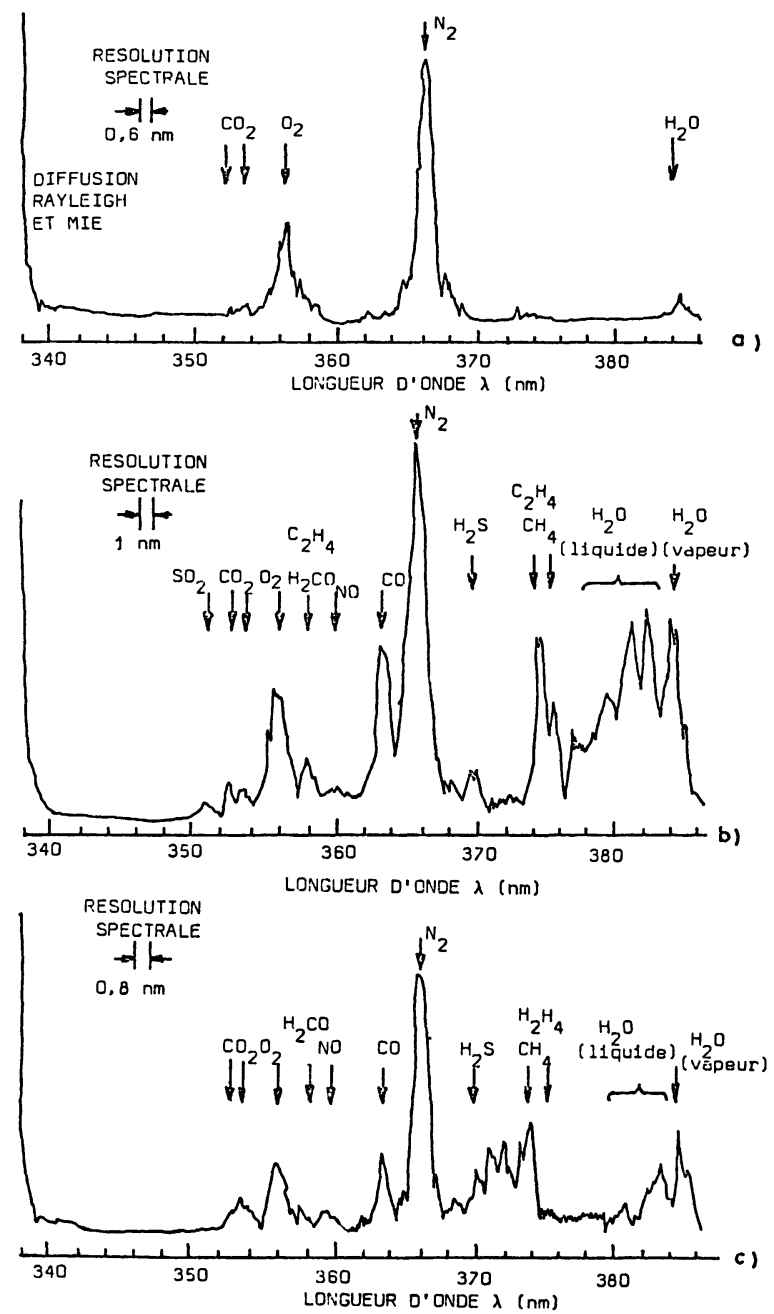

Fig. 6. - Distribution spectrale des raies Raman de différents polluants industriels (d'après [57]).

L'utilisation de cette méthode reste donc limitée aux courtes portées et les principales applications concernent la surveillance de la pollution industrielle. Des

concentrations de quelques ppm (correspondant à un rapport de mélange de $10^{-6}$ ) sont ainsi mesurables avec des portées de quelques centaines de mètres et des temps d'intégration de l'ordre de la minute qui permettent de suivre l'évolution spatiale d'atmosphères très polluées au voisinage, par exemple, de cheminées d'usines [32] (Fig. 6). L'application à la surveillance de la pollution en atmosphère urbaine est plus difficile, car les concentrations caractéristiques sont plus faibles, de l'ordre du ppb $\left(\sim 10^{-9}\right)$.

Ce processus a également été utilisé pour la mesure de la concentration atmosphérique totale à partir de la raie de l'azote moléculaire, ce qui permet avec un laser émettant à une seule longueur d'onde d'éliminer la contribution due aux aérosols, indiscernable comme nous l'avons vu précédemment dans le cas d'une diffusion élastique : la portée reste cependant limitée à la basse stratosphère [33].

Une amélioration importante de l'efficacité peut être obtenue en se plaçant au voisinage d'une raie de résonance de la molécule (Fig. $3 c$ ) : c'est la diffusion Raman résonnante qui correspond à une augmentation de la section efficace dę 3 à 6 ordres de grandeur. Elle se distingue de la fluorescence par le temps d'interaction lumière-molécule $\left(\sim 10^{-14} \mathrm{~s}\right.$ [34]) et peut donc s'appliquer dans les basses couches de l'atmosphère [35]. Cette méthode augmente donc les possibilités d'application de la diffusion Raman, mais devient spécifique du constituant étudié et nécessite alors l'utilisation de laser à fréquence accordable, de façon à se placer d'une façon très bien définie au voisinage de la raie de résonance. Pour préciser l'ordre de grandeur des seuils de détectabilité accessibles par ces différentes techniques de diffusion, dont les valeurs caractéristiques sont résumées dans le tableau I, nous avons porté sur la figure 7 en fonction de l'altitude les concentrations minimum mesurables en $\mathrm{cm}^{-3}$ avec une précision de $10 \%$ pour une énergie laser totale émise de $100 \mathrm{~J}$, une surface réceptrice de $1 \mathrm{~m}^{2}$ et un rendement optique total de $10^{-2}$. En outre, le tableau II donne une indication de seuils minimum de détectabilité par diffusion Raman de quelques constituants

Tableau I. - Processus de diffusion applicables à la télédétection laser des constituants atmosphériques.

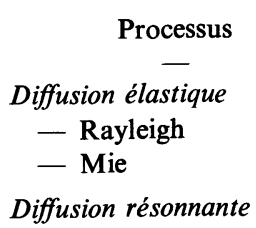

Fluorescence

Diffusion Raman

Diffusion Raman résonnante

\footnotetext{
Caractéristiques

$\lambda$ réception $=\lambda$ émission $\sigma \sim 10^{-28} \mathrm{~cm}^{2}\left(\lambda^{-4}\right)$$$
\sigma \sim 10^{-10} \mathrm{~cm}^{2}
$$$$
\lambda \text { réception }=\lambda \text { émission }
$$$$
\sigma \sim 10^{-12} \mathrm{~cm}^{2} \text { (atomes) }
$$$$
\sigma \sim 10^{-17} \mathrm{~cm}^{2} \text { (molécules) }
$$$$
\lambda \text { réception } \neq \lambda \text { émission }
$$$$
\sigma \sim 10^{-18} \mathrm{~cm}^{2}
$$$$
\lambda \text { réception } \neq \lambda \text { émission }
$$$$
\sigma \sim 10^{-29} \mathrm{~cm}^{2}
$$$$
\lambda \text { réception } \neq \lambda \text { émission }
$$$$
\sigma \sim 10^{-25} \mathrm{~cm}^{2}
$$ 


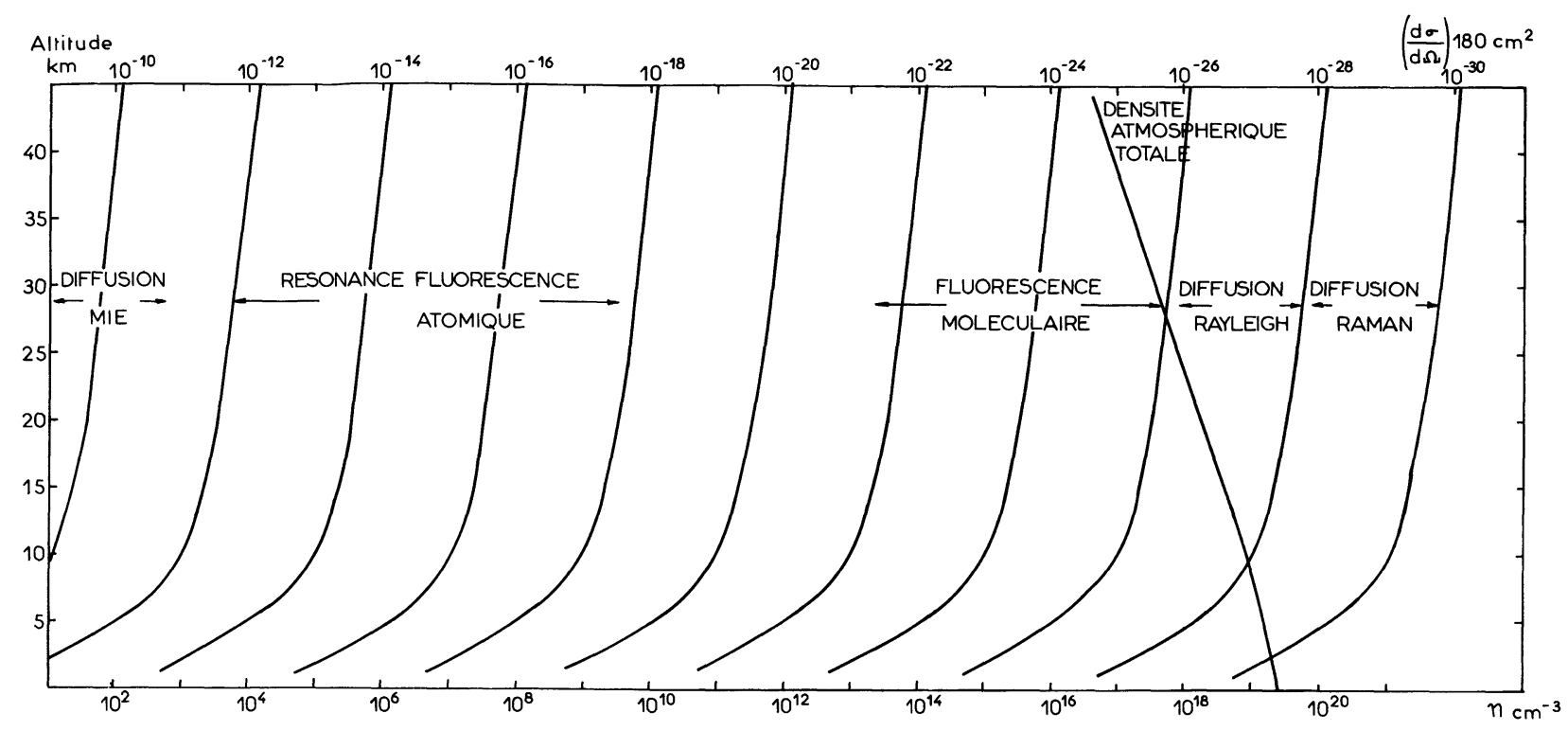

Fig. 7. - Concentration minimum, en fonction de l'altitude, mesurable par rétrodiffusion avec une précision de $10 \%$ en 100 tirs laser (énergie $1 \mathrm{~J}$, surface réceptrice $1 \mathrm{~m}^{2}$, rendement optique $10^{-2}$ ).

de la basse stratosphère. Pour considérer ces résultats avec optimisme, donc se convaincre de l'avenir de ces méthodes, on peut constater que les processus les plus efficaces sont en général associés aux constituants les moins abondants.

Tableau II. - Sensibilité des mesures par diffusion Raman.

$\begin{array}{cc}\text { Constituant } & \begin{array}{c}\text { Concentration relative } \\ \text { minimum détectable }\left(^{*}\right)\end{array} \\ \mathrm{SO}_{2} & \sim 1 \mathrm{ppm} \\ \mathrm{N}_{2} \mathrm{O} & \sim 1 \mathrm{ppm} \\ \mathrm{H}_{2} \mathrm{O} & \sim 50 \mathrm{ppm} \\ \mathrm{CO}_{2} & \sim 100 \mathrm{ppm}\end{array}$

(*) (Laser Rubis SH $(347,2 \mathrm{~nm}) 1 \mathrm{~J}$, portée $5 \mathrm{~km}$, résolution $1 \mathrm{~km}$, surface réceptrice $0,5 \mathrm{~m}^{2}$, intégration sur 100 tirs lasers.)

3. Sondage par absorption différentielle. - Il est bien évident que cette méthode ne peut s'appliquer que dans les domaines spectraux où les molécules atmosphériques possèdent des bandes d'absorption, ce qui conduit à priori à distinguer dans le domaine des longueurs d'onde optiques.

- Les transitions électroniques situées généralement dans l'ultraviolet en 250 et $400 \mathrm{~nm}$ pour les molécules atmosphériques $\left(\mathrm{O}_{3}, \mathrm{NO}_{2}, \mathrm{SO}_{2}, \mathrm{ClO} \ldots\right)$. Les variations avec la longueur d'onde des sections efficaces d'absorption $\left(\sim 10^{-19} \mathrm{~cm}^{2}\right)$ sont suffisamment lentes pour permettre l'emploi de raies laser larges d'environ $0,1 \mathrm{~nm}$, l'intervalle spectral séparant une région absorbante d'une région sans absorption étant de l'ordre d'une dizaine de nanomètres.

Notons également dans le proche infrarouge $(\lambda \sim 760-900 \mathrm{~nm})$ la présence de bandes d'absorption de la vapeur d'eau et de l'oxygène moléculaire (ban- des A) correspondant à des transitions interdites et donc à des sections efficaces d'absorption plus faibles $\left(\neq 10^{-23} \mathrm{~cm}^{2}\right)[36]$.

- Les transitions de vibration pure dans l'infrarouge entre 2,5 et $20 \mu \mathrm{m}$. Les molécules d'intérêt atmosphérique présentent en général un tel spectre de vibration pur, à l'exception des composés majoritaires homopolaires azote et oxygène moléculaires. Les raies de vibration diffèrent d'une molécule à l'autre quant à leur intensité, leur position spectrale et leur forme ce qui permet en principe de déterminer pour chaque constituant un intervalle spectral caractéristique. Cependant les risques d'interférences entre les différents composés restent importants [37] et il est nécessaire de faire une étude spectroscopique fine du domaine spectral étudié pour interpréter les résultats sans ambiguiité [38]. Les largeurs spectrales utilisées pour les lasers sont ici de l'ordre de $0,1 \mathrm{MHz}$ et correspondent donc à une résolution spectrale de $10^{8}-10^{9}$ supérieure de 4 à 5 ordres de grandeur à celles utilisées dans l'ultraviolet [39].

Si l'on utilise pour les mesures par absorption différentielle la diffusion Rayleigh ou Mie par les particules atmosphériques, on peut conserver la résolution spatiale du système lidar. En effet, à partir de l'équation (1) on peut calculer la puissance moyenne reçue pour une altitude $z$ et une longueur d'onde $\lambda_{1}$ :

$P\left(\lambda_{1}, 0, z\right)=P_{0}\left(\lambda_{1}\right) G\left(\lambda_{1}, z\right) \tau_{n}\left(\lambda_{1}, z\right) \mathrm{e}^{-2 \tau_{a}\left(\lambda_{1}, 0, z\right)}$.

Dans la mesure où le facteur $G\left(\lambda_{1}, z\right)$ peut se décomposer sous la forme $G_{0}\left(\lambda_{1}\right) G_{1}(z)$, on peut écrire

$$
\begin{aligned}
R= & \frac{P\left(\lambda_{1}, 0, z+\mathrm{d} z\right)}{P\left(\lambda_{1}, 0, z\right)} \frac{P\left(\lambda_{2}, 0, z\right)}{P\left(\lambda_{2}, 0, z+\mathrm{d} z\right)}= \\
= & \mathrm{e}^{-2\left(\tau_{\mathbf{a}}\left(\lambda_{1}, z, z+\mathrm{d} z\right)-\tau_{\mathbf{a}}\left(\lambda_{2}, z, z+\mathrm{d} z\right)\right)}
\end{aligned}
$$


c'est-à-dire que, si les sections efficaces $\sigma_{\mathrm{a}}\left(\lambda_{1}\right)$ et $\sigma_{\mathrm{a}}\left(\lambda_{2}\right)$ du composé étudié sont connues, la concentration moyenne $\bar{n}_{\mathrm{a}}$ de ce composé est donnée à partir du rapport $R$ de quatre puissances mesurées par

$n_{\mathrm{a}}(z)=\frac{\log 1 / R}{2\left(\sigma_{\mathrm{a}}\left(\lambda_{1}\right)-\sigma_{\mathrm{a}}\left(\lambda_{2}\right)\right) \mathrm{d} z}$.

Cette mesure ne nécessite donc aucun étalonnage absolu de l'intensité laser, puisque la comparaison se fait directement entre deux intervalles d'altitude consécutifs. Etant donné la limite de sensibilité et de précision que l'on peut espérer pour les chaînes électroniques classiques $\left(\sim 10^{-3}\right)$, cette méthode permet donc de mesurer des épaisseurs optiques de l'ordre de $10^{-2}$ à $10^{-3}$ ou supérieures. Cette limitation implique que pour un constituant donné et donc des valeurs fixées et optimisées des sections efficaces, la résolution spatiale $\mathrm{d} z$ soit déterminée par le seuil de détectabilité et donc plus le constituant sera minoritaire, plus l'on perdra en résolution sur son profil. Pour des valeurs classiques des sections efficaces utilisables dans l'ultraviolet $\left(\sim 10^{-18}\right.$ à $\left.10^{-19} \mathrm{~cm}^{2}\right)$ et une résolution de $1 \mathrm{~km}$, on ne peut ainsi espérer détecter que des constituants dont la concentration reste supérieure à $10^{10} \mathrm{~cm}^{-3}$, ce qui correspond dans la troposphère à un rapport de mélange de $10^{-9}(\sim \mathrm{ppb})$ et dans la stratosphère à un rapport de $10^{-7}(\sim 0,1 \mathrm{ppm})$. Ces limites restent cependant très inférieures à celles de la spectroscopie classique ou des méthodes chimiques de détection, et la technique lidar par absorption

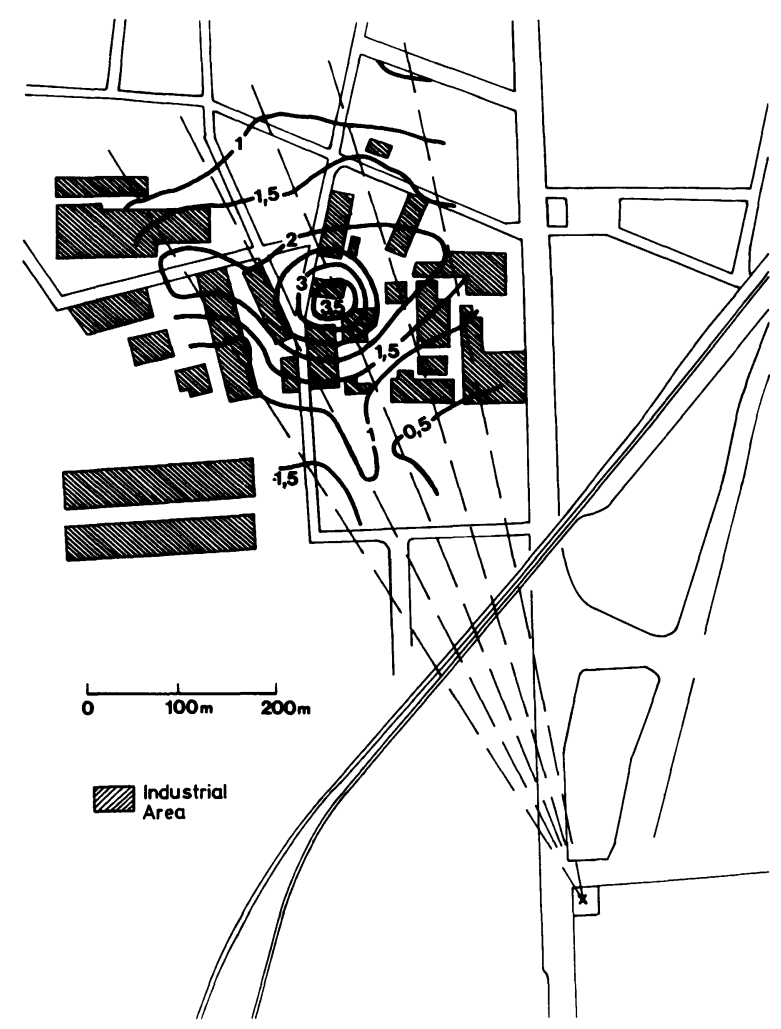

Fig. 8. - Isodensités (ppm) de $\mathrm{NO}_{2}$ autour d'une cheminée d'usine mesurées par absorption différentielle (d'après [42]). différentielle constitue donc un moyen très puissant d'étude de la basse atmosphère. La précision de la mesure est, comme nous l'avons vu, fonction de la puissance moyenne rétrodiffusée et donc, compte tenu de la variation en $\lambda^{-4}$, de la section efficace Rayleigh, on voit que pour une puissance laser moyenne constante $\left(\sim \lambda^{-1}\right)$ la perte d'efficacité entre le domaine ultraviolet $(\lambda \sim 0,3 \mu)$ et l'infrarouge $(\lambda \sim 10 \mu)$ atteint $4 \times 10^{4}$.

Les sondages verticaux par rétrodiffusion sont donc restés pour l'instant essentiellement limités au domaine ultraviolet. La source laser est alors un laser à fréquence accordable obtenu par doublage en fréquence d'un laser à colorant (R $6 \mathrm{G})$ pompé par flashs [40]. Les énergies utilisables sont de l'ordre de 50 à $100 \mathrm{~mJ}$ pour une largeur spectrale de $0,1 \mathrm{~nm}$ et un domaine d'utilisation continu allant de $290 \mathrm{~nm}$ à $320 \mathrm{~nm}$, qui permet de couvrir l'essentiel des transitions électroniques. Cette méthode a jusqu'à présent été employée avec succès dans la troposphère $\left(\mathrm{SO}_{2}, \mathrm{O}_{3}[41]\right)\left(\mathrm{NO}_{2}\right.$ [42]) (Fig. 8) et dans la stratosphère pour l'ozone [43] (Fig. 9), bien que la précision des mesures reste encore assez faible.

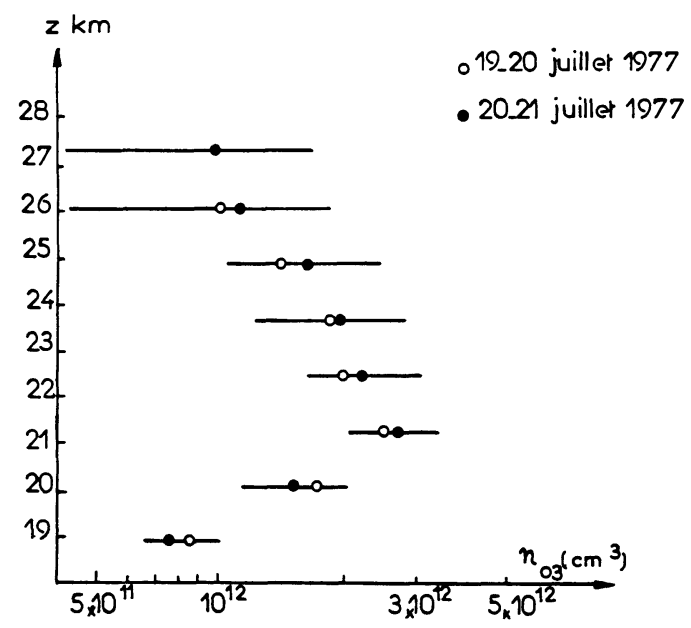

Fig. 9. - Profils verticaux d'ozone stratosphérique mesurés par absorption différentielle à partir du sol (d'après [43]).

Une des limitations principales de ce domaine de longueur d'onde est l'importance de l'extinction atmosphérique, conséquence évidente de l'augmentation cherchée de la diffusion. L'interprétation des mesures en termes de composants minoritaires d'épaisseur optique faible $\left(\sim 10^{-2}\right.$ à $\left.10^{-3}\right)$, nécessite donc une connaissance précise de la concentration atmosphérique ou de l'apparition d'aérosols diffusants. On peut cependant penser que la mise au point des lasers à excimères devrait permettre d'utiliser cette technique pour des mesures de routine de l'ozone troposphérique ou pour la surveillance de la pollution.

Dans le proche infrarouge $(\lambda \sim 700 \mathrm{~nm})$, des mesures de la vapeur d'eau ont été effectuées avec une portée verticale de 1 à $2 \mathrm{~km}$ [44]. L'utilisation de longueurs d'onde plus élevées : $820 \mathrm{~nm}$ et $940 \mathrm{~nm}$ 
devraient permettre d'effectuer des mesures à plus haute altitude $(\sim 7$ à $8 \mathrm{~km})$ si l'on s'affranchit de l'absorption prépondérante des premières centaines de mètres (sites en altitude) [45]. Les énergies laser nécessaires pour de telles mesures sont de l'ordre du Joule pour des largeurs spectrales de quelques pm, et donc de nouveaux développements de lasers à colorant dans le proche infrarouge doivent être poursuivis.

Dans le domaine infrarouge, étant donné la faible efficacité des processus de diffusion, les mesures par absorption différentielle ont surtout été effectuées grâce à un système rétro-réflecteur permettant de réfléchir totalement l'énergie laser émise (Fig. 2). Il s'agit donc essentiellement dans ce cas, de mesure d'épaisseur optique intégrée au niveau du sol qui impliquent donc la perte de la résolution spatiale associée au lidar. Ces expériences utilisent principalement des systèmes émetteurs continus [46] (diodes lasers, oscillateurs paramétriques, laser à $\mathrm{CO}$ ou $\mathrm{CO}_{2}$ ) et ont permis la détection d'un grand nombre de polluants gazeux $\left(\mathrm{NO}_{2}, \mathrm{CH}_{4}, \mathrm{CO}, \mathrm{NO}, \mathrm{O}_{3}, \mathrm{C}_{2} \mathrm{H}_{4}\right.$, fréons 11 et 12) [47, 48, 49].

L'utilisation à partir d'une plate-forme embarquée sur ballon, d'un laser à $\mathrm{CO}$ accordable en fréquence (Spin flip Raman) [50] a également permis une mesure absolue du monoxyde d'azote NO dans la stratosphère $(28 \mathrm{~km})$. L'emploi dans ce domaine de longueurs d'onde des techniques de détection cohérente (hétérodyne) devrait permettre de gagner de 2 à 3 ordres de grandeur sur la sensibilité des mesures. Elles n'ont jusqu'à présent été appliquées que sur la source solaire pour la mesure de l'ozone stratosphérique et troposphérique [51], le profil en altitude de ce constituant étant déduit par inversion, de la forme spectrale de la raie d'absorption à partir du profil vertical de température supposé connu. L'extension de ces techniques, non seulement pour des mesures intégrées [52], mais aussi pour des systèmes pulsés, devrait permettre de conjuguer la très grande sensibilité spectrale des mesures infrarouges avec la résolution spatiale du système lidar [53]. Une des applications très précieuse de ces techniques serait alors la mesure dans les premiers kilomètres de l'atmosphère des flux d'un certain nombre de constituants troposphériques $\left(\mathrm{H}_{2} \mathrm{O}, \mathrm{CH}_{4}, \mathrm{~N}_{2} \mathrm{O}\right.$, hydrocarbures...), dont on sait qu'ils jouent un rôle prépondérant dans la photochimie

Tableau III. - Sensibilité des mesures par absorption différentielle dans l'ultraviolet pour quelques constituants atmosphériques.

$\begin{array}{ccc}\text { Constituant } & \text { Longueur d'onde } & \begin{array}{c}\text { Concentration relative } \\ \text { minimum détectable }\left(^{*}\right)\end{array} \\ - & - & - \\ \mathrm{SO}_{2} & 0,30 \mu & 2 \mathrm{ppb} \\ \mathrm{NO}_{2} & 0,45 \mu & 20 \mathrm{ppb} \\ \mathrm{O}_{3} & 0,30 \mu & 4 \mathrm{ppb}\end{array}$

$\left(^{*}\right)$ (Laser de $100 \mathrm{~mJ}$, surface réceptrice $0,5 \mathrm{~m}^{2}$, portée de $2 \mathrm{~km}$, résolution $500 \mathrm{~m}$, intégration sur 1000 tirs lasers.) de la stratosphère [54] et pour lesquels les conditions à la limite inférieure restent très mal connues.

A titre d'exemple, nous avons porté dans les tableaux III et IV les sensibilités calculées de mesures par absorption différentielle dans l'ultraviolet et dans l'infrarouge pour quelques constituants atmosphériques. Cette liste ne saurait évidemment être exhaustive et l'application à de nombreux autres constituants atmosphériques a déjà été étudiée de façon précise [55].

Tableau IV. - Sensibilité des mesures par absorption différentielle dans l'infrarouge pour quelques constituants atmosphériques.

$\begin{array}{ccc}\text { Constituant } & \text { Longueur d'onde } & \begin{array}{c}\text { Concentration relative } \\ \text { minimum détectable }\left(^{*}\right)\end{array} \\ - & -\overline{\mathrm{O}_{3}} & 4 \overline{\mathrm{ppb}} \\ \mathrm{NH}_{3} & 9,48 \mu & 0.5 \mathrm{ppb} \\ \mathrm{SO}_{2} & 8,7 \mu & 7 \mathrm{ppb}\end{array}$

(*) (Laser $\mathrm{CO}_{2} 1 \mathrm{~J}$, surface réceptrice $1 \mathrm{~m}^{2}$, portée $5 \mathrm{~km}$, résolution $1 \mathrm{~km}$, intégration sur 10 tirs lasers.)

4. Conclusion. - L'utilisation des lasers pour l'étude de l'atmosphère terrestre constitue un nouveau domaine de recherche important de l'optique atmosphérique. La possibilité d'obtenir de façon continue des profils verticaux de constituants mineurs semble aujourd'hui largement démontrée dans toutes les zones d'altitude (troposphère, stratosphère et mésosphère). La généralisation et l'extension en altitude de méthodes très sensibles comme la diffusion Raman ou l'absorption différentielle, jusqu'à présent limitées aux basses couches troposphériques, sont pour l'essentiel tributaires de l'augmentation de la puissance des lasers existants, augmentation qui ne peut en aucun cas se faire au détriment des qualités spatiales et spectrales du faisceau.

Dans ce domaine, l'amélioration de la densité spectrale d'énergie par réduction de la largeur d'émission permettra, grâce à l'utilisation de traceurs passifs (oxygène moléculaire, aérosols dans les basses couches, sodium en haute altitude), de mesurer des paramètres essentiels de l'atmosphère comme la température, la pression ou les vents moyens. L'utilisation dans les basses couches du lidar, conjuguée à celles d'autres sondeurs du type radar ou sodar qui permettent d'accéder à d'autres paramètres, constitue un outil extrêmement puissant pour la compréhension des phénomènes thermodynamiques et des processus d'échange de la basse atmosphère.

Enfin l'emploi de plates-formes embarquées (avions, ballons ou satellites) tel qu'il est envisagé dans un avenir très proche [56] doit permettre, en s'affranchissant de l'absorption des basses couches, d'ouvrir de nouveaux domaines de longueurs d'onde et d'effectuer des mesures in situ avec une très bonne sensibilité et sans perturbation du milieu ambiant. 


\section{Bibliographie}

[1] Elterman, L., J. Geophys. Res. 56 (1951), 509.

[2] Pettifer, R. E. W., J. Atm. Terr. Phys. 37 (1975) 669.

[3] Blamont, J. E., Chanin, M. L., Megie, G., Ann. Geophys. 28 (1972) 833.

[4] Huffaker, R. M., Atmospheric Technology (NCAR) 1974, 71.

[5] Mason, J. B., Appl. Opt. 14 (1975) 76.

[6] Smullin, L. D., Fiocco, G., Nature 194 (1962) 1267.

[7] Kent, G. S., Sandland, P., Wright, R. W. H., J. Appl. Meteor. 10 (1971) 443.

[8] Kent, G. S., Keenliside, W., J. Atmo. Sci. 31 (1974) 1409.

[9] McCormick, M. P., Electro-Optics International Conference Brighton, England, 1971.

[10] Fiocco, G., Grams, G., J. Atmo. Sci. 21 (1964) 323.

[11] Fernald, F. G., Schuster, B. G., Frush, C., Opt. Quant. Electron. 7 (1975) 141.

[12] Hirono, M., Fujiwara, M., Can. J. Chem. 52 (1974) 1560.

[13] Russel, P. B., Hake, R. D., J. Atmo. Sci. 34 (1977) 163.

[14] Noonkester, V. R., Jensen, D. R., Richter, J. H., Viezee, W., Collis, R. T. H., J. Appl. Meteorol. 13(1974) 249.

[15] Russel, P. B., Uthe, E. E., Ludwig, F. L., Shaw, N. A., J. Geophys. Res. 79 (1974) 36, 5555.

[16] DeIRmendijan, D., Electromagnetic scattering on spherical polydispersions (American Elsever New York) 1969.

[17] Carnuth, W., Jäger, H., Littfass, M., Reiter, R., Proc. Conf. Opto Electronics Laser (Munich) 1977 p. 728.

[18] Pal, S. R., Carswell, A. I., Appl. Opt. 12 (1973) 1530.

[19] Bowman, M. R., Gibson, A. J., Sandford, M. C. W., Nature 221 (1969) 456

[20] Blamont, J. E., Chanin, M. L., Megie, G., C.R. Hebd. Séan. Acad. Sci. 274 (1972) 93

[21] Schuler, C. J., Pike, C. T., Miranda, H. A., Appl. Opt. 10 (1971) 1689.

[22] Gibson, A. J., J. Phys. E. (Sci. Instrum.) 2 (1969) 802.

[23] Loth, C., Megie, G., J. Phys. E. (Sci. Instrum.) 7 (1974) 80.

[24] Maeda, M., Okada, T., Uchino, O., Miyazoe, Y., Japan J. Appl. Phys. 15 (1976), 1731.

[25] Loth, C., Meyer, Y. H., Bos, F., Opt. Commun. 16 (1976) 310.

[26] Megie, G., Bos, F., Chanin, M. L., Blamont, J. E., Planet. Space Sci. 26 (1978) 27.

[27] Megie, G., Blamont, J. E., Planet. Space Sci. 25 (1977) 1093.

[28] Wang, C. C., Davis Jr. L. I., Phys. Rev. Lett. 32 (1974) 349.

[29] Tucker, A. W., Petersen, A. B., Birnbaum, M., Appl. Opt. 12 (1973) 2036

[30] CoOney, J. A., Appl. Phys. Lett. 12 (1968) 40.

[31] Koybayasi, T., Ibana, M., Proc. IEEE 58 (1970) 1568.

[32] Melfi, S. H., Brumfield, M. L., Storey, Jr. R. W., Appl. Phys. Lett. 22 (1973) 402.

[33] Garvey, M. J., Kent, G. S., Nature 248 (1974) 124.
[34] Behringer, J., J. Raman Spectro. 2 (1974) 275.

[35] Penney, C. M., Proceedings 8th International Laser Radar Conference, Philadelphia, 1977.

[36] Burch, D. E., GrynnaK, D. A., Appl. Opt. 8 (1969) 1493.

[37] Shewchun, J., Garside, B. K., Ballik, E. A., Kwan, C. C. V., Elsherbiny, M. M., Hogenkamp, G., Kazadjian, A., Appl. Opt. 15 (1976) 340.

[38] Hanst, P. L., Appl. Spectrosc. 24 (1970) 161

[39] Hinkley, E. D., Opt. Quant. Electro. 8(1976) 155.

[40] Hirth, A., Vollrath, K., Allain, J. Y., Opt. Commun. 20 (1977) 350.

[41] Grant, W. B., Hake, R. D., J. Appl. Phys. 46 (1975), p. 3019.

[42] Rothe, K. W., Brinkman, U., Walther, H., Appl. Phys. 4 (1974) 181.

[43] Megie, G., Allain, J. Y., Chanin, M. L., Blamont, J. E., Nature, 270 (1977) 5635, 329.

[44] Schotland, R. M., Proc. 14th Weather Radar Conference, Tucson, Ariz. 1971.

[45] Browell, E. V., Brumfield, M. L., Siviter, J. H., Northam, G. B., Wilkerson, T. D., McIlrath, T. J., Proc. 8th International Laser Radar Conference, Philadelphie, 1977.

[46] Hinkley, E. D., Opto-Electron 4 (1972) 69.

[47] Hinkley, E. D., Kelley, P. L., Science 171 (1971) 635.

[48] Montgomery, G. P., Hill, J. C., J. Opt. Soc. Am., 65 (1975) 579.

[49] Kuhl, J., Spitschan, H., Opt. Commun. 13 (1975) 6.

[50] Patel, C. K. N., Burkhardt, E. G., Lambert, C. A., Science 184 (1974) 1173

[51] Menzies, R. T., Seals, R. K., Science 197 (1977) 4310, p. 1275.

[52] Menzies, R. T., Opt. Engin. 17 (1978) 44.

[53] Murray, E. R., Opt. Engin. 16 (1977) 284.

[54] COVOS Rapport final Paris 1976.

[55] Collis, R. T. H., Russel, P. B., Laser Monitoring of the Atmosphere ed. by E. D. Hinkley (Springer Verlag, Berlin) 1976, p. 70.

[56] Hinkley, E. D., Ku, R. T., Kelley, P. L., Laser Monitoring of the Atmosphere ed. by E. D. Hinkley (Springer Verlag, Berlin) 1976 p. 237.

[57] Ibana, H., Laser Monitoring of the Atmosphere ed. by E. D. Hinkley (Springer Verlag, Berlin) 1976 p. 155.

[58] Collis, R. T. H., Hake, R. D., Russel, P. B., Bowhill, S. A., Opt. Engineering 17 (1978) 1, 23.

[59] Jegou, J. P., Blamont, J. E., Chanin, M. L., Megie, G., Communication privée, 1978.

[60] Pourny, J. C., Renaud, D., Orszag, A., A publier dans Applied Optics (1979).

[61] Orszag, A., Zaharla, R., De Valence, Y., C.R. Hebd. Séan. Acad. Sci. 267 (1968) p. 237. 